\title{
An unusual case of Acanthamoeba peritonitis in a malnourished patient on continuous ambulatory peritoneal dialysis (CAPD)
}

\author{
R.Tilak, ${ }^{1}$ R.G. Singh, ${ }^{2}$ I.A. Wani, ${ }^{2}$ A. Parekh, ${ }^{2}$ J. Prakash, ${ }^{2}$ and Usha. ${ }^{3}$ \\ ${ }^{1}$ Department of Microbiology, Institute of Medical Sciences, Banaras Hindu University, Varanasi-221 005 India. \\ ${ }^{2}$ Nephrology, Institute of Medical Sciences, Banaras Hindu University, Varanasi-221 005 India. \\ ${ }^{3}$ Pathology, Institute of Medical Sciences, Banaras Hindu University, Varanasi-221 005 India.
}

\begin{abstract}
An unusual case of peritonitis in a 61-year-old patient is reported where culture for bacteria and fungi were negative. Acanthamoeba was isolated and the patient was treated with Ceftazidine, Cefazolin, Levofloxacin, Fluconazole and Rifampicin with regular haemodialytic support. The patient was completely cured of the infection and continuous ambulatory peritoneal dialysis (CAPD) fluid became clear after 2 weeks of treatment. Diagnosis and treatment of Acanthamoeba infections are difficult due to the rarity of the infections, lack of familiarity of most clinicians with disease syndromes, and limitations of therapeutics options. Even an experienced microbiologist can easily mistake the amoebae in ascitic fluid for peritoneal macrophages or lymphocytes.

Key Words: Continuous Ambulatory Peritoneal Dialysis (CAPD), Acanthamoeba, Peritonitis.
\end{abstract}

J Infect Developing Countries 2008; 2(2):146-148.

Received 19 October 2007 - Accepted 28 February 2008.

Copyright (C) 2007 Tilak et al. This is an open access article distributed under the Creative Commons Attribution License, which permits unrestricted use, distribution, and reproduction in any medium, provided the original work is properly cited.

\section{Introduction}

The process of continuous ambulatory peritoneal dialysis (CAPD) has provided a useful, relatively inexpensive, and safe alternative procedure for patients with end stage renal disease. Infectious peritonitis, however, has limited the acceptance of this technique [1-3]. Peritonitis results from decreased phagocytic efficiency with depressed phagocytosis and bactericidal capacity of peritoneal macrophages. Free-living amoebae of the genus Acanthamoeba are the causative agents of many infections in immunocompromised patients and may be life threatening. Extraneural infective foci in the skin, paranasal area, or lungs are possible points of access for amoebae [4]. The most important finding in our patient was made by direct demonstration of the amoebae in the ascitic fluid and also by culture.

\section{Case Report}

A 61-year-old male suffering from non-insulin dependent diabetes mellitus for the past nine years presented with generalized weakness and swelling. For the first six years the patient received oral hypoglycemic agents and subsequently was given insulin for controlling hyperglycemia. He was diagnosed with hypertension three years ago. Anasarca and stage $\mathrm{V}$ diabetes chronic kidney disease (diabetes nephropathy) were diagnosed two years ago. In February 2006, the patient was started on chronic ambulatory peritoneal dialysis and was doing well on 4 exchanges per day. Four months later, the patient was hospitalized with a history of low-grade intermittent fever, mild abdominal pain, cloudy effluent dialysate, nausea and vomiting for one month, and altered sensorium for five days. The patient had stopped peritoneal dialysis 10 days before hospitalization.

On examination the patient was irritable with aggressive behavior, and not oriented in time, place and person. The patient was febrile $\left(102^{\circ} \mathrm{F}\right)$, with pallor and pitting edema in both lower limbs. His pulse rate was 110 per minute and blood pressure was $150 / 90 \mathrm{~mm} \mathrm{Hg}$ in the right upper arm supine position.

The patient's chest and cardiac examination was unremarkable. His abdominal examination revealed distended abdomen with generalized tenderness, mild guarding and normal bowel sounds. The exit site and tunnel of the peritoneal 
dialysis catheter were healthy without any drainage. The patient's investigations revealed hemoglobin of $4.8 \mathrm{gm} / \mathrm{dl}$, total leukocyte count of 17,000 cells $/ \mathrm{mm} 3$ with the differential count showing $64 \%$ neutrophils, 33\% lymphocytes, $2 \%$ eosinophils and $1 \%$ monocytes. His peripheral blood film revealed normocytic, normochromic anaemia with no hemoparasites. His random blood glucose levels were $146 \mathrm{mg} / \mathrm{dl}$ and kidney function tests revealed urea of $214 \mathrm{mg} / \mathrm{dl}, \mathrm{S}$. creatinine of $13 \mathrm{mg} / \mathrm{dl}$ with normal electrolytes $(\mathrm{Na}+=140$ $\left.\mathrm{meq} / \mathrm{L}, \mathrm{K}_{+}=4.5 \mathrm{meq} / \mathrm{L}\right)$. His liver function tests revealed bilirubin of $0.9 \mathrm{mg} / \mathrm{dl}$, SGOT of 26 units, SGPT of 34 , Serum protein of $4.6 \mathrm{gm} / \mathrm{dl}$ and albumin of $1.3 \mathrm{gm} / \mathrm{dl}$.

Both blood and urine cultures were found to be sterile. Peritoneal dialysis effluent showed 165 cells/cmm with $81 \%$ lymphocytes and showed negative results on Gram's stain and Ziehl Neelsen stain.

Empirically, Ceftazidime $1 \mathrm{gm}$ and Cefazolin 1 gm intraperitoneally 3 times a day and intravenous Levofloxacin 500mg daily were started immediately. Peritoneal dialysis was stopped due to poor outflow and the patient was given thrice weekly hemodialysis. The patient's mental status improved and he regained consciousness but continued with fever and abdominal symptoms over the next 10 to 15 days. His dialysis effluent wet mount examination showed the presence of free-living amoebae. The motile forms presented the characteristic morphological features of spherical shape, having abundant cytoplasm and nuclei with prominent nucleoli consistent with the morphology of Acanthamoeba species (Figure 1). A specimen inoculated on non-nutrient agar (NNA) covered with a dense lawn of the Escherichia coli yielded Acanthamoeba species.

The patient was continued on thrice weekly hemodialysis, Ceftazidine and Levofloxacin and in addition was treated with Rifampicin $600 \mathrm{mg}$ daily and Fluconazole $150 \mathrm{mg}$ daily. After 2 weeks of treatment, the patient's fever settled, abdominal signs and symptoms disappeared, and his appetite improved. Repeat peritoneal fluid examination was found negative for Acanthamoeba $s p$. and peritoneal dialysis was restarted with clear effluent dialysate.
Figure 1. Acanthamoeba species found in the patient's peritoneal fluid.

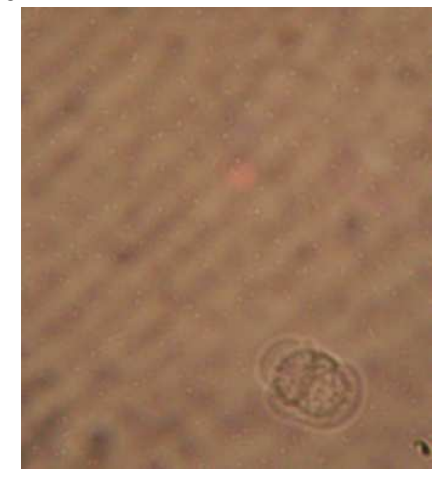

\section{Discussion}

Peritonitis remains the major concern in patients on CAPD; however, with improved technology the incidence of peritonitis has decreased to less than one episode in 24 months. Patients usually present with abdominal pain, nausea and vomiting, fever, chills and constipation or diarrhoea. Cloudy peritoneal fluid is seen in almost all patients with peritonitis, as well as abdominal tenderness, increased body temperature, and increased blood leucocytes $[5,6]$.

Fungal peritonitis occurs in $1 \%$ to $10 \%$ of cases, whereas in $5 \%$ to $20 \%$ of cases no organism can be isolated. We are reporting a case of a patient suffering diabetic nephropathy on CAPD due to Acanthamoeba species. This parasite is an opportunistic free-living amoeba capable of infecting persons with depressed immune systems. Acanthamoeba species are causative agents of granulomatous amoebic encephalitis and amoebic keratitis, and have been associated with cutaneous lesions and sinusitis [2]. Immunocompromised individuals, including AIDS patients, are particularly susceptible to infections with the parasite. The life cycle of the amoeba includes an active feeding trophozoite stage and dominant cyst stage with no flagellate stage. Trophozoites are infective forms and are believed to gain entry via the lower respiratory tract and ulcerated or broken skin.

If properly treated, typical cases of peritonitis have a mortality rate of about $<10 \%$ in otherwise healthy patients; this figure rises to about $40 \%$ in elderly patients. If untreated, generalized peritonitis is almost always fatal.

In our case, Acanthamoeba was isolated from peritoneal dialysis effluent in a patient with diabetic nephropathy on CAPD. The parasite was identified 
by light microscopy based on its characteristic appearances. It has not been previously reported as a cause of peritonitis in patients on CAPD. Another interesting feature of our case was the successful treatment of peritonitis using Rifamipicin $600 \mathrm{mg}$ and Fluconazole $150 \mathrm{mg}$ daily along with empiric antibiotics.

In conclusion, Acanthamoeba is a rare cause of peritonitis on CAPD. This case is even more unusual in view of successful treatment with Fluconazole and Rifampicin. It should be considered in culture negative peritonitis. Delayed diagnosis of Acanthamoeba peritonitis often results in death. Clinical awareness of this entity may lead to early diagnosis and proper treatment.

\section{References}

1. Marciano-Cabral F, Cabral G (2003).Acanthamoeba spp. as agents of disease in Humans. Clinical microbiological reviews 16: (2) 273-307.

2. Avon G, Amsterdam D (1992) Microbiological aspects of peritonitis associated with continuous ambulatory peritoneal dialysis. Clin. Microbiol Rev.5:36-48.

3. Li PK, Law MC, Chow KM, Szeto CC, Cheng YL, Wong TY, Leung CB, Wang AY, Lui SF, Yu AW (2002)
Comparison of clinical outcome and ease of handling in two double-bag systems in continuous ambulatory peritoneal dialysis - a prospective randomized controlled multi-center study. Am J Kidney Dis. 40:373-380.

4. Martinez AJ, GS Visvesvara (1997) Free living amphizoic and opportunistic amoebas. Brain Patho7: 583-598.

5. Gahrmani N, Gorban-Brennan N, Kliger AS, Finkelstein FO (1995) Infection rates in end-stage renal disease patients treated with CCPD and CAPD using the UltraBag system. adv Perit Dial. 11: 164-167.

6. Moneteon F, Correa-Rotter R, Paniagua R, Amato D, Elena Hurtado M, Medina JL, Salcedo RM, Garcia E, Mario M, Julio K, Roberto V, Alfonso R, Maria AS, John M (1998) Prevention of peritonitis with disconnect systems in CAPD: A randomized controlled trial. The Mexican Nephrology Collaborative Study Group. Kidney Int. 54: 2123-2138.

Corresponding Author: Ragini Tilak, Department of Microbiology, Institute of Medical Sciences, Banaras Hindu University, Varanasi-221 005 India, Email: raginijain29@rediffmail.com

Conflict of interest: No conflict of interest is declared. 